\title{
Methods of drainage and transfer of rainwater
}

\author{
Robert Malmur ${ }^{1, *}$ \\ ${ }^{1}$ Institute of Environmental Engineering, ul. Brzeźnicka 60a, 42-200 Częstochowa, \\ Poland
}

\begin{abstract}
With frequent heavy rainfalls in summer in Poland and fast-melting snow in spring leading to flooding of sewage systems (due to excessive filling levels in water receivers or difficulties with temporary retention of the excess stormwater), a variety of systems are being developed to facilitate transfer of the stormwater to water receivers. Outflow of the excess stormwater is usually ensured by the use of gravitational outflow collectors that connect stormwater drains with waterways. The transfer occurs during intensive precipitation, when the excess wastewater overflows through stormwater drains and is transferred directly to water receivers in order to relieve wastewater treatment plants or to minimize diameters of sewers. These systems are useful wherever the filling levels in waterways are not very high or the sewerage system is located relatively high with respect to the water receiver i.e. outflow collector is located on a steep slope. In such cases, the stormwater that flows through a waterway cannot be returned to the outflow collector. If the gravitational flow is impossible due to e.g. the excessive filling level of water receiver, stormwater can be transferred by means of a variety of modern solutions, such as retention and transfer reservoirs. These reservoirs are supposed to ensure partial retention of the excess stormwater and transfer of this water to water receivers, either gravitationally or forced mechanically, depending on the filling level in the waterway. The transfer reservoirs for the stormwater presented in this paper might be successfully used in modernization of current sewage systems, ensuring the reliability of operation and a more effective wastewater transfer than the systems used to date. All the reservoirs of this type are characterized by similar design and function and guarantee that the wastewater might be transferred regardless of the conditions in the water receiver.
\end{abstract}

\section{Introduction}

Various countries all over the world use different solutions to the problem of wastewater transfer. There are plethora of solutions in the form of stationary pumping stations and portable pumping stations (e.g. in China). However, the outflow of excess wastewater from precipitation occurs gravitationally through outflow collectors that connect stormwater with waterways $[1,2]$.

A flap return valve is often installed at the outlet from the outflow collectors to prevent from flooding of the protected areas. Flap valves prevent from the backflow in the

\footnotetext{
*Corresponding author: rmalmur@ is.pcz.czest.pl
} 
stormwater system caused by high levels of water in rivers [3]. However, in practice, these valves are not always used or are left without adequate supervision and maintenance. Consequently, poor state of repair usually limits efficient operation of these valves [4].

Unsecured outlets of outflow collectors or inefficiently operated flap valves have led in recent years to numerous instances of flooding in residential areas of many cities in Poland.

Another problem is observed in areas directly adjacent to rivers or artificial dams where raised water levels are much higher than land elevation. Such depressed areas with respect to constant filling on the other side of the embankment are deprived of natural conditions for water outflow from their drainage basin.

In such cases, building adequate stations for transfer of water from water receiver is critical. In practice, the pumping stations are located at the points of wastewater discharge.

If the surface water (also including stormwater from precipitation) is released directly to the water receiver (river), the protection in the form of flap valves installed on the river side of the embankment should be monitored and maintained at regular intervals. However, they become inaccessible in case of raised river levels [2].

The filling levels that make gravitational outflow impossible lead to the need for building a system of transfer of wastewater from the protected area to the water receiver.

The inefficient and often unreliable solutions of wastewater transfer have become the reason for starting the research on new and more efficient solutions to this problem.

Retention and transfer reservoirs for stormwater took over the function of the effective transfer of wastewater from precipitation to the water receiver. Wastewater transfer occurs if gravitational outflow is inhibited periodically or permanently.

Periodical inhibition of the transfer of wastewater from precipitation can be caused by high filling levels in the river, which are also observed in the period of intensive precipitation. Furthermore, permanent inhibition is observed if the dewatered areas are located in the depression with respect to the filling levels in the receiver due to the accumulation of water $[5,6]$.

\section{The transfer reservoir for stormwater (PLUVIUS type)}

\subsection{Characteristics and the design of the transfer reservoir}

The PLUVIUS type stormwater transfer reservoir [5] consists of a drainage chamber, a pumping unit and a tower chamber, the height of which is at least equal to the height of the crest of the flood embankment of a waterway. Connected by a outflow collector, the waterway and the tower chamber form a system of communicating vessels, which means that they have the same filling levels, especially during suddenly rising levels.

In the bottom zone, the tower chamber is connected to the drainage chamber by a transit channel with a valve installed the end of the channel. The valve located in a transit channel can be of any design, for example a gate valve or a manually or mechanically operated flap. With the simple design of the reservoir, it is advantageous to use the valve as a self-opening flap, which would allow for a gravitational flow from the drainage chamber to the tower chamber.

The pumping unit is located above the drainage chamber. It is connected to the tower chamber by a pumping pipe.

Such a solution enables the gravitational outflow of wastewater to the water receiver when the watercourse filling levels are lower than the collector filling level or the trench supplying sewage from the catchment area to the drainage chamber. The drainage chamber allows for the gravitational flow of wastewater through the transit channel, a tower chamber and an outflow collector (under a flood embankment) directly to the river bed. 
In the case of higher filling levels of the watercourse, the connection of the drainage chamber to the watercourse is cut off by a flap valve whereas the wastewater filling the drainage chamber is discharged by the pumping unit into the tower chamber.

In such a transfer reservoir, it is recommended that the pumping unit should contain at least two pumps whose total volumetric flow rate should be greater than the calculated maximum wastewater inflow. Wastewater level sensors are installed in the drainage chamber allowing for controlling the system of pumps. The control system for the pumps consists of at least four sensors located at different heights.

a).

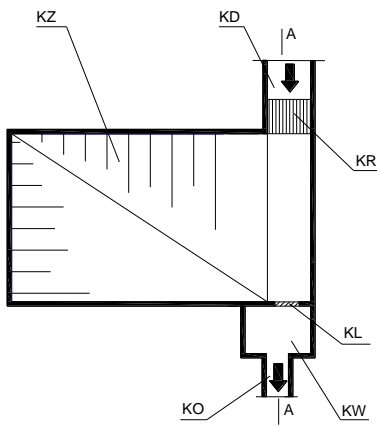

b).

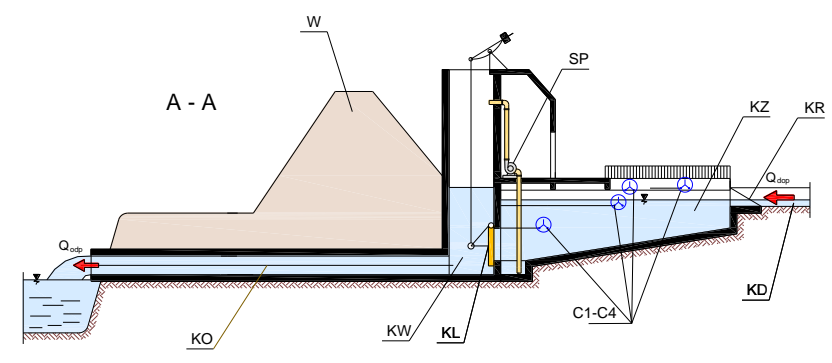

Fig. 1. Diagram of the PLUVIUS-P type transfer reservoir: a) top view, b) A-A cross-section; $\mathrm{KD}$ - inflow collector, $\mathrm{KR}$ - grating, KZ - drainage chamber, SP - pumping unit, C1-C4 - wastewater level sensors, $\mathrm{KL}$ - non-return flap, $\mathrm{KW}$ - tower chamber, $\mathrm{KO}$ - outflow collector, $\mathrm{W}$ - flood embankment.

The wastewater is supplied to the reservoir by means of a collector or an inflow trench (KD), with a grating (KR) installed at the end of the trench (Fig. 1). The transfer reservoir consists mainly of a drainage chamber $(\mathrm{KZ})$ and a tower chamber $(\mathrm{KW})$, connected to the bottom zone by a transit channel. The height of the tower chamber $(\mathrm{KW})$ is greater than the height of the watercourse crest. There is a flap valve (KL) installed in the opening of the transit channel. The valve is unidirectional, self-operating, and directs the flow towards the tower chamber $(\mathrm{KW})$. The drainage collector $(\mathrm{KO})$, which is located at the bottom, connects the watercourse and the tower chamber $(\mathrm{KW})$. With specific dimensions and stop $\log$ closures, the tower chamber (KW) allows for installing, operating and possible repair of the valve (KL).

The function of the drainage chamber (KZ) is not to retain wastewater, but to temporarily stop it when gravity drainage is impossible. The volume of such a chamber results from the assumed minimum time of its complete filling at the calculated maximum inflow and lack of possibility of gravitational outflow to the watercourse. The bottom of the drainage chamber (KZ) is shaped so that during the period of gravitational outflow of wastewater to the water receiver, the transit flow does not cause filling of the entire bottom surface of the chamber. This can be achieved, for example, by means of a through cavity that provides a flow rate corresponding to the calculated maximum inflow.

The discharge of wastewater from the drainage chamber (KZ) to the tower chamber $(\mathrm{KW})$ in the period when gravity drainage is impossible occurs through a pumping unit. Such a unit consists of two pumps (ZP), whose total volumetric flow rate is about $10 \%$ higher than the calculated maximum $\mathrm{Q}_{\text {dop max }}$ flow rate occurring in a given catchment, and four sewage level sensors (C1), (C2), (C3) and (C4). These sensors are located at different heights in the sin drainage chamber $(\mathrm{KZ})$. 
Excessively long sewage retention time in the drainage chamber (KZ) may cause the sewage to rot. Therefore it seems advisable to install an additional sensor on the sewage supply channel $(\mathrm{KD})$ to the reservoir, which will switch on the pump even if the wastewater inflow to the reservoir disappears, in order to transfer the remaining wastewater volume in the drainage chamber.

The pressure line of the pumps (PT) reaches almost the bottom of the drainage chamber $(\mathrm{KZ})$ and is led to the tower chamber $(\mathrm{KW})$.

\section{The transfer reservoir for stormwater (GEMINUS-ET type)}

\subsection{Characteristics and the design of the transfer reservoir}

The transfer reservoir GEMINUS-ET type has the flow-through chamber (KP1) linked in a top zone with the inlet channel (KD) and the overflow chamber (KP2) [7]. The flow-through chamber (KP1) and the overflow chamber (KP2) are separated by a barrier (PR) with overflow (Fig. 2a). The edge of overflow is located lower than the top point of the inlet channel (KD) the outline of. The flow-through chamber (KP1) is linked through the check valve (KL1) with the gas-proof storage chamber (KZ1). Both the storage chambers (KZ1, KZ2) through the following check valve (KL3, KL4) are combined with the tower chamber $(\mathrm{KW})$. The tower chamber $(\mathrm{KW})$, of height greater than ordinate of water levee is combined with a water receiver indirectly through the outlet channel (KO). The check valves (KL3, KL4) work automatically as they are opened by hydrostatic pressure.

In a ceiling of the storage chambers (KZ1, KZ2) there is a pipeline (P1) linking together with the shut-off valves (Z1, Z1a) this zone with the air-compressors (SP1) or the atmosphere. One the shut-off valve (Z1) cuts the storage chamber (KZ1) off the line of the air-compressor, whereas the other one cuts this chamber from the atmosphere, and similar thing happens in another the storage chamber (Fig. 2b). Air pipes are joined together with an extra line through the shut-off valve. Air pipes and a special control of the shut-off valves enables to use the pressure of compressed air caught in the previously emptied the storage chamber to a partial evacuation of the other chamber without using the air-compressor.

With low and average states of filling a water receiver, there are certain conditions preserved for gravitational outflow of the storm water to the receiver which flows with the help of the inlet channel (KD) to the transfer reservoir.

With high states of filling in a water receiver when gravitational outflow of sewages is not possible, storm water which flow in with the help of the inlet channel (KD) to the flow-through chamber (KP1) of the reservoir through the opened the check valve (KL1) fills the first storage chamber (KZ1). While filling process, appropriate the shut-off valves (Z1a) placed on the air pipe (P1) are opened. This way they enable the air outflow from the storage chamber (KZ1) to the atmosphere.

The process of filling the first storage chamber (KZ1) is finished when its completely filled with storm water and the flow chamber (KP1) is filled to the level of overflow (PR). Accomplishing such filling states cause switching the first compressor (SP1) on and cutting off appropriate the shut-off valves (Z1a) placed on air pipe (P1). That contributes to the storage chamber's cutting off which is filled with storm water from the atmosphere. That's how the process of making the storage chamber empty is started.

The increase of air pressure forced in to the empty chamber by the compressor causes the storm water outflow to the tower chamber $(\mathrm{KW})$. After the process of making the first 
chamber empty the compressor (SP1) is switched off and appropriate the shut-off valves (Z1) closed. Because of this process compressed air is kept in empty chamber.

The process of making the first storage chamber (KZ1) empty as well as the process of filling the second storage chamber (KZ2) starts simultaneously. Because at this stage of chamber's functioning the check valve (KL1) closes the inlet to the first storage chamber (KZ1), the storm water which flows into the reservoir through overflow fills the overflow (PR) chamber and then fills the second storage chamber (KZ2). While the second storage chamber (KZ2) is filled, appropriate the shut-off valves placed on air pipe are opened. This way they enable the air outflow from the storage chamber (KZ2) to the atmosphere. Filling the second chamber (KZ2) and the overflow chamber (KP2) happens simultaneously.

a).

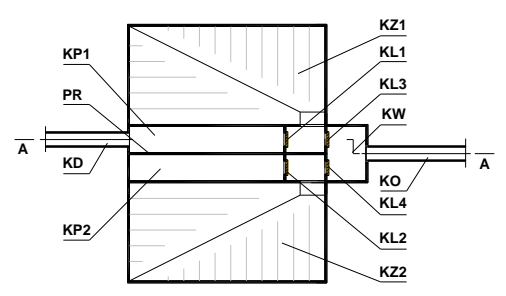

b).

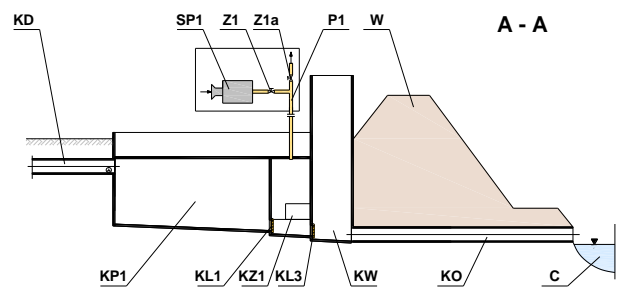

Fig. 2. Example solution transfer reservoir GEMINUS-ET: a) top view, b) cross section A-A; KZ1, KZ2 - storage chambers, KP1 - flow-through chamber, KP2 - overflow chamber, $\mathrm{KW}$ - tower chamber, KD - inlet channel, KO - outlet channel, KL1-KL4 - check valves, PR - partition wall with weir, W - flood embankment, C - water receiver, Z1, Z1a - shut-off valves, SP1 - pressure compressor, P1 - air pressure pipe.

Complete the second storage chamber (KZ2) with liquid is possible thanks to maintaining compressed air left in the first storage chamber (KZ1) after making it empty.

The complete filling of the second storage chamber (KZ2) will make the shut-off valves close and this chamber cut off the atmosphere. Simultaneously opening of the shut-off valve on the line which links both air pipe will enable compressed air-flow from the first storage chamber (KZ1) (which was stopped after it had been emptied) to the second chamber (KZ2). The process of partial emptying of the storage chamber by compressed air is started. The pressure of compressed air is balanced in both cut off from the atmosphere the storage chambers.

The increase of air pressure in the second storage chamber (KZ2) causes the storm water flow from this chamber to the tower chamber $(\mathrm{KW})$. The equalizing of air pressure in both cut off atmosphere the storage chambers leads to partial emptying the second chamber (KZ2). In the next part, for the complete emptying of this chamber from the storm water, it is necessary to switch off the second air-compressor with simultaneous opening and closing certain the shut-off valves.

After the complete emptying of the second storage chamber (KZ2) the air-compressor is switched off and simultaneously closed a proper the shut-off valve, so the compressed air is preserved in this chamber.

While pneumatic emptying of the second storage chamber the storm water that flows up to the receiver fills the first storage chamber again, which is always opened into the atmosphere while the air-compressor is working. This rule is compulsory in the opposite situation, that is, when the pneumatic emptying of the first storage chamber appears through the air-compressor.

Accomplishment of the complete level of filling in the first storage chamber (KZ1) will cause its cut off from the atmosphere. Simultaneously the proper shut-off valve will open 
which enables the flow of compressed air from the second storage chamber (KZ2) to the first chamber (KZ1). This is the way of beginning a repeated process of the partial emptying of the storage chamber through the balancing the air pressure in both separated from the atmosphere the storage chambers.

The increase of the air pressure in the first storage chamber (KZ1) will cause the flow of the storm water from this chamber to the tower chamber (KW). After the finishing of the process of the air pressure the first air-compressor is switched on again and the proper shut-off valves are opened and closed. The air-compressor empties only partially filled the storage chamber till its complete emptying. In the meantime the storm water in flowing to the receiver is taken off by the second storage chamber (KZ2). Such alternate filling and emptying of the chambers of the reservoir allows to transfer the storm water flowing into it to the water receiver regardless of the fact if it is full or not.

\section{The transfer reservoir for stormwater type GEMINI}

\subsection{Characteristics and the design of the transfer reservoir}

The transfer reservoir for stormwater discussed in this study can be built near channels of water receivers where the wastewater is supposed to be transferred to. The reservoir is supposed to be built near the flood embankment or embedded in the embankment $[7,10]$.

Wastewater to the reservoirs are transported to the flow chamber through outflow collector and discharged from the tower chamber to a water receiver through an outflow collector (Fig. 3). The pump chambers are equipped in one-way flap valves, with the flow oriented towards the tower chamber. The flow chamber is connected with the pump chambers through pumping channels build in lower part of the flow chamber and in front part of pump chambers. Pump chambers contain cut-off valves that prevent from air access in particular cases. The reservoir is also equipped in electrical pump-compressor unit located over the pump chamber and controlled by means of sewage level sensors build in the pump chambers and the flow chamber. The pumping units comprise of air-tight pump chamber and pump-compressor unit connected by means of suction and pumping channel through the roof to the pump chamber. The pump channels are connected to the hydraulic system between one-way flap valves build in the bottom zone with the flow oriented towards the tower chamber, whereas the pumping channels are connected with the flow chamber.

An essential structural feature of the reservoir is that the flow chamber is built below the pump chambers. With this solution, sewage are transported to the flow chamber and then, through the pumping channels, to the pump chambers. Depending on the sewage level in the water receiver, sewage from pump chambers are transferred gravitationally or forced by pressure.

Sewage flows to the reservoir through the inflow collector (KD) to the flow chamber (KP) (Fig. 3a). There is a small cavity at the end of the flow chamber (KP), where pumping channels (KT1 and KT2) are connected. These channels are used to connect the flow chamber (KP) with pump chambers (KZ1, KZ2) (Fig. 3b) through the wholes made in the front part of these chambers. The top of the pumping channels (KT1, KT2) reaches almost to the roof of the pump chambers (KZ1, KZ2). Pumping channels (KT1, KT2) have cut-off valves $(Z 1, Z 2)$, build in the upper part of the flow chamber (KP). Pump chambers (KZ1, KZ2) are connected with the tower chamber (KW) through one-way flap valves (KL1, KL2) which operate automatically, oriented with the flow towards the tower chamber $(\mathrm{KW})$. 

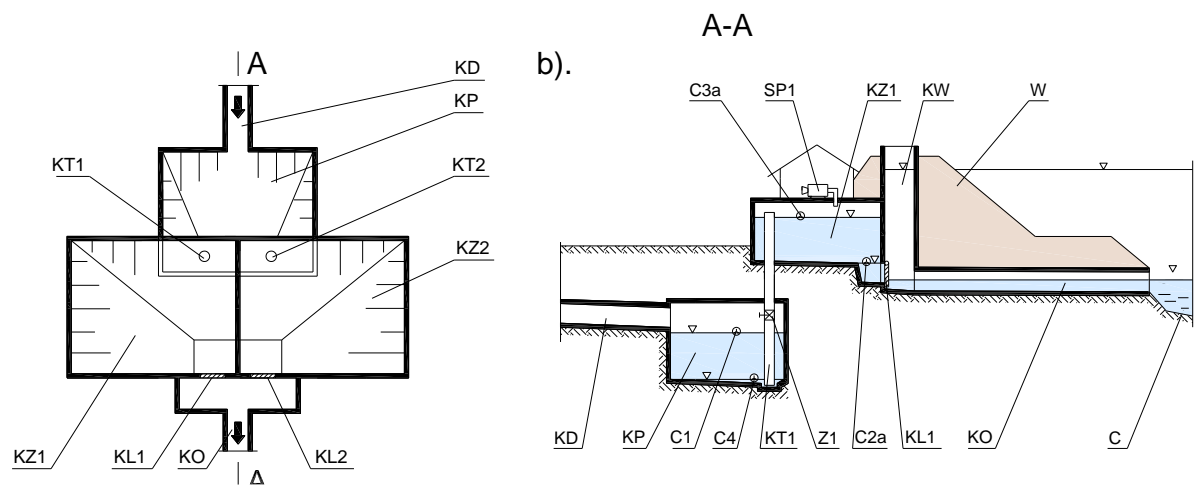

Fig. 3. Transfer reservoir of stormwater, a) top view, b) cross section A-A; KZ1, KZ2 - pump chambers, $\mathrm{KP}$ - flow chamber, $\mathrm{KW}$ - tower chamber, $\mathrm{KD}$ - inflow collector, $\mathrm{KO}$ - outflow collector, KL1,KL2 - check valves, W - flood embankment, C - water receiver, Z1 - shut-off valve, KT1, KT2 - pumping channel, C1-C4 - sewage level sensors, SP1 - pump-compressor unit.

The roof of the pump chamber (KZ1, KZ2) contains two pump-compressor units (SP1, SP2), connected through pipes with above-roof space of these chambers. The pump-compressor units (SP1, SP2) are of similar design and their flow rate equals at least the equivalent maximum inflow $\mathrm{Q}_{\text {dopmax }}$ present in a particular drainage basin. The pump chambers (KZ1, KZ2) are built as air-tight, they have similar volume and are designed next to each other.

The tower chamber $(\mathrm{KW})$ is higher than the other chambers and reaches the height at least equal to the height of the crest of the flood embankment (W) of the waterway (C). Sewage from the tower chamber (KW) flows to the waterway (C) through an outflow collector (KO). The outflow collector (KO) runs along the bottom of the waterway (C) under the flood embankment (W), whereas the outlet of the collector is located under the water surface in the water receiver $(\mathrm{C})$.

Sewage level sensors $(\mathrm{C} 1, \mathrm{C} 2 \mathrm{a}, \mathrm{C} 2 \mathrm{~b}, \mathrm{C} 3 \mathrm{a}, \mathrm{C} 3 \mathrm{~b}, \mathrm{C} 4)$ that control operation of pump-compressor units (SP1, SP2) are built in pump chambers (KZ1, KZ2) and flow chamber (KP). The (C1) sensor is installed in the flow chamber (KP) at the height of several centimetres below the bottom of the inflow collector (KD), whereas the (C4) sensor is located at the bottom of the chamber, at the height of ca. $5 \mathrm{~cm}$ over the lower part of the pumping channels (KT1, KT2). Sensors (C2a, C2b, C3a and C3b) are built in the pumping chambers (KZ1, KZ2). Lower sensors (C2a, C2b) are located at the upper level of the flap chamber (KL1, KL2), whereas the upper sensors (C3a, C3b) are located at the height of ca. $5 \mathrm{~cm}$ below the upper part of the pumping channels (KT1, KT2).

\section{Conclusions}

Present solutions for stormwater transfer to water receivers used in the most of the cities of Poland are obsolete, unreliable and, apart from the extension, need urgent modernization. The unreliability of these systems is manifested primarily with frequent flooding in the sewage system in the case of elevated levels in water receivers.

Regardless of the size of the drainage basin and the used sewage system, transfer reservoirs should be viewed today as one of the most basic components in contemporary systems of the transfer of the stormwater to water receivers. Building such facilities involves considerable investments while proper operation is possible only if appropriate 
design and high-quality suction and pumping units are used as they consume considerable amounts of electricity to transfer the wastewater from lower to higher level or to transfer the wastewater up and to the location that ensures its gravitational outflow at the same time.

The transfer reservoirs for the stormwater presented in this paper might be successfully used in modernization of current sewage systems, ensuring the reliability of operation and a more effective wastewater transfer than the systems used to date. All the reservoirs of this type are characterized by similar design and function and guarantee that the wastewater might be transferred regardless of the conditions in the water receiver. An essential feature of these reservoirs is the use of the effective method to control suction and pumping units.

Using the reservoir needs a thorough analysis of the data about the drainage basin, sewage system, equipment and facilities involved while taking into consideration minimum costs of implementation of a particular investment [8].

Adequate determination of the sufficient retention capacity is of key importance to the process of design with consideration of their effect on both reliability and costs of investment and operation.

Temporal and spatial irregularity of real precipitation offers many opportunities connected with adjustment of control equipment to the current hydraulic load of the system, both in the reservoir filling and discharge phases.

The retention and transfer reservoir type GEMINI presented in this study is one of the proposals for the solutions to the problem of periodical flooding of urban areas. The system of air suction and pumping used in this reservoir ensures more reliable operation than the system of wastewater pumps installed since it does not go into direct contact with wastewater that contains mechanical pollutants and are often chemically aggressive.

This scientific study was financed within the research project BS-PB 401-301/17.

\section{References}

1. M. Mrowiec, Effective dimensioning and dynamic control of sewage reservoirs (Monograph 171, Published by University of Czestochowa, 2009)

2. W. Geiger, H. Dreiseitl, New methods of draining storm water (Projprzem, Warszawa 1999)

3. J. Łomotowski, Rain water and extreme events (Monograph, Seidel-Przywecki, 2011)

4. W. Błaszczyk, M. Roman, H. Stamatello, Sewerege (Arkady, Warszawa 1974)

5. R. Malmur, A. Kisiel, M. Mrowiec, J. Kisiel, CzT 18, 105 (2008)

6. M. Mrowiec, T. Kamizela, M. Kowalczyk, Environ. Prot. Eng. 35, 2 (2009)

7. R. Malmur, Rocz. Ochr. Sr. 13 (2011)

8. M. Mrowiec, A. Kisiel, R. Malmur, J. Kisiel, Pol. J. Environ. Stud. 16, 2A, part III (2007) 